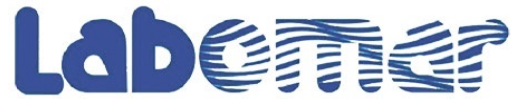

Arquivos de Ciências do Mar

\section{CHECKLIST, RICHNESS OF COMMON NAMES AND CONSERVATION ISSUES OF MARINE FISHES LANDED IN COMMERCIAL FISHERIES OF ESPÍRITO SANTO STATE, BRAZILIAN CENTRAL COAST}

\author{
Lista das espécies, diversidade de nomes comuns e status de \\ conservação dos peixes marinhos capturados na pesca comercial \\ no estado do Espírito Santo, costa central brasileira
}

\author{
Joelson Musiello-Fernandes ${ }^{1}$, Ryan Andrades ${ }^{2}$, Caio Ribeiro Pimentel ${ }^{3}$, \\ Michelle Sequine Bolzan ${ }^{4}$, Andreia Schwingel ${ }^{5}$, Maurício Hostim-Silva ${ }^{6}$ \\ ${ }^{1}$ Pós-doutorando do Programa de Pós-Graduação em Oceanografia Ambiental, (bolsista Capes - código 001), \\ Universidade Federal do Espírito Santo, Joelson.pesca@gmail.com \\ 2 Laboratório de Ictiologia, Programa de Pós-Graduação em Oceanografia Ambiental, \\ Universidade Federal do Espírito Santo, ryanandrades@gmail.com \\ ${ }^{3}$ Doutorando do Programa de Pós-Graduação em Oceanografia Ambiental, bolsista da \\ Fundação de Amparo à Pesquisa do Espírito Santo (Fapes), Laboratório de Ictiologia, \\ Departamento de Oceanografia e Ecologia, pimentelcr@yahoo.com.br \\ ${ }^{4}$ Escritório de Projetos, Fundação Espírito Santense de Tecnologia, Universidade Federal \\ do Espírito Santo, bolzan.ms@gmail.com \\ ${ }^{5}$ Laboratório de Recursos Pesqueiros Artesanais e Modelagem Ecológica, Instituto de Oceanografia, \\ Universidade Federal do Rio Grande (FURG), Programa de Pós-Graduação em \\ Biologia de Ambientes Aquáticos Continentais. deiaschwingel@gmail.com \\ ${ }^{6}$ Laboratório de Ecologia de Peixes Marinhos LEPMAR/UFES, Universidade Federal do \\ Espírito Santo. mhostim@gmail.com
}

\begin{abstract}
The aim of this manuscript is to provide the first checklist of marine commercial fish species landed in Espírito Santo state (Brazilian central coast), with associated richness of common names and conservation status. The data collection took place under the Fisheries Monitoring Program, throughout the state of Espírito Santo. Altogether 22 fishing landing ports were monitored from May 2011 to August 2012. A total of 91 fish species from 39 families were identified. The richness of common names of fishery resources in Espírito Santo showed an average of two common names per species.
\end{abstract}

Keywords: folk names, commercial landings, fisheries monitoring, Espírito Santo, Brazil.

\section{RESUMO}

O objetivo deste artigo é fornecer a primeira lista de espécies de peixes marinhos com os nomes comuns e status de conservação, desembarcadas no estado do Espírito Santo (ES), costa central do

Recebido em: 03/10/2019

Aprovado em: 05/05/2020

Publicado em: 30/08/2020 
Brasil. A coleta de dados ocorreu no âmbito do Programa de Estatística Pesqueira, em todo o estado do Espírito Santo. O monitoramento aconteceu em 22 portos de desembarque de pesca, no período de maio de 2011 a agosto de 2012. Ao todo, foram identificadas 91 espécies de peixes pertencentes a 39 famílias. A diversidade de nomes comuns dos recursos pesqueiros no Espírito Santo apresentou média de dois nomes comuns por espécie.

Palavras-chave: nomes populares, desembarque pesqueiro, estatística pesqueira, Espírito Santo, Brasil.

\section{INTRODUCTION}

Marine resources have been subjected to several impacts, such as the increase of biodiversity losses driven by fishing (Worm et al., 2006), which have impacted the per capita consumption of fishery resources (Garcia, 2010), catch rates and several commercial stocks (Pauly et al., 2002; FAO, 2014) thereby increasing the debate on fisheries sustainability in the literature (Coulthard, 2012; Jacquet \& Pauly, 2008).

Historically, managing and monitoring small-scale tropical fisheries (e.g. Brazil) is a challenging task due to the heterogeneity of fishing gear, landing territoriality and, mainly, the scarcity of data of fishery statistics (Defeo \& Castilla, 2005; Begossi, 2014). In this sense, some local fisheries and catches of commercial species can be overestimated if the richness of attributed common fish names is not considered. For instance, multiple common names can be attributed to one fish species by local fishers, while in the vicinity one common name can fit a couple of species (Freire \& Pauly, 2005; Freire \& Carvalho-Filho, 2009). In Brazil, the richness of common names can be ascribed to a cultural and linguistic richness inserted in a huge coastal territory $(>8,000 \mathrm{~km})$. Thus, the knowledge of richness of common names of marine exploited resources in a region is fundamental to a better managing decisions and fishery estimates. The first step to evaluate and support effective management fisheries measures comprises the knowledge of fishery dynamics and correct identification of target species (Freire \& Pauly, 2005; Anticamara et al., 2011).

The last update of Brazilian national production of fisheries resources reported a fishery catch around 1.431.974,4 ton/year (MPA, 2011), of these 13.5\% (553.670,0 ton/year) are from marine fisheries; while in the Espírito Santo state (ES) it was approximately 12,000 ton/year (Hostim-Silva \& Soares, 2013). The Espírito Santo state covers only 5\% of the Brazilian coastline, however, recent studies indicated important pelagic and coastal resources being exploited and landed along their coast (Martins, Olavo \& Costa, 2005; Hostim-Silva \& Soares, 2013; Musiello-Fernandes et al., 2018). The aim of this manuscript is to provide the first checklist of marine fish species landed in the state of Espírito Santo, central coast of Brazil, and the common names associated to fish species.

\section{MATERIALS AND METHODS}

\section{Study area}

The Espírito Santo state $\left(18^{\circ} 35^{\prime} \mathrm{S}\right.$ to $\left.21^{\circ} 31^{\prime} \mathrm{S}\right)$ is located in the lower portion of Brazilian central coast (Martins, Olavo \& Costa, 2005). This region presents a variation in continental shelf width with higher extension in north part, reaching up to $246 \mathrm{~km}$ (Martins \& Coutinho, 1981). The continental shelf margin and oceanic region adjacent is marked by 
the presence of Vitória-Trindade submarine ridge $\left(20^{\circ}-21^{\circ} \mathrm{S}\right)$. The circulation pattern of the region is dominated by the oligotrophic waters of the Brazilian Current (Campos et al., 2000; Martins; Olavo \& Costa, 2005).

Twenty two fishing communities and villages along the Espírito Santo coast were monitored by the Fishery Monitoring Program of Espírito Santo (Programa de Estatística Pesqueira do Espírito Santo) (Hostim-Silva \& Soares, 2013). Each fishing community was monitored daily by a local member that interviewed the fishermen about fishery production and dynamics. Concomitantly to fish landings, the identification of fish species was made using the classic and current identification guides, such as Figueiredo (1977), Figueiredo and Menezes (1978, 1980, 2000), Menezes and Figueiredo (1980, 1985), Cervigón et al. (1992), Carvalho-Filho (1999), Carpenter (2002). National common names (BRA common names) cited in this paper were based on Carvalho-Filho (1999), Freire and Carvalho-Filho (2009), Froese and Pauly (2019). The state common names used by local fishermen (ES common names) were obtained by interviews applied during the fish landings. The species conservation status was determined following the Brazilian Red Book of the Endangered Species (ICMBio, 2018).

\section{RESULTS AND DISCUSSION}

Altogether 91 fish species from 39 families were identified (Table I). The four most speciose families were Scombridae (11\%), followed by Sciaenidae $(8 \%)$, Carangidae $(7 \%)$ and Lutjanidae (7\%). This dominance of scombrids, mainly of the genus Thunnus was related to hand line/hook-and-line and longline fleet that operate in the south of Espírito Santo state (Itaipava and Guarapari region), which exploits mainly tunas, dolphinfishes and billfishes as main target resources. According to Martins et al. (2005), the artisanal Itaipava fleet comprises small fishing boats (11-15 meters) that fishing generally in the "Bacia de Campos" $\left(21^{\circ}-23^{\circ} \mathrm{S}\right)$, a region of the continental shelf that shelter an important oil drilling platforms field.

The richness of ES common names was, in average, two names per species, which is lower than reported for Brazilian reef fishes (seven common names per species; Freire \& Carvalho-Filho, 2009). On the other hand, 56.1\% of fish species reported here were associated to two or more common names (e.g. Robalo, Vaquara and Cação), which hamper the accuracy of catch estimates of these fishing resources. The synonyms (different common names for one species) and homonyms (same common name for more than one species) can influence commercial catch statistics of specific resources, as seen for the catfishes Genidens genidens, Aspistor quadris cutis and Cathorops spixii reported by Freire and Pauly (2005), which catch values could be grouped into the "Bagre" common name. In this case, the inventory effort of specific exploited resources may be improved with the exact identification of a target resource or the determination of prevalence of some species in catch data.

Additionally, highly target species usually had their common names in relation to size of catch, such as "Dourado" and "Palombeta" and "Atum galha amarela" and "Vaquara", which refers to larger and smaller individuals, respectively, of Coryphaena hippurus and Thunnus albacares. In other case, the high richness of common names seems to refer a natural variation of brazilian common name, as with "Ariocó" and "Biquara". This variation was observed in small fishery villages of Espírito Santo.

Among the fish landed, Ginglymostoma cirratum is classified as Vulnerable (ICMBio, 2018). The main threat to this species in the Espírito Santo coast is the spear-fishing, 
however, the G. cirratum fisheries recorded in this study were caught by gill netting and hook-and-line activities, being the latter performed in adjacent grounds of the Marine Protected Area "Parque Nacional Marinho dos Abrolhos". Others species classified as Vulnerable were Mycteroperca bonaci, Epinephelus morio and Lopholatilus villarii. Also, the record of the endemic endangered Scarus trispinosus reinforce the need for understanding of fisheries dynamics and target resources in order to develop tools that help the coastal fishery management.

The checklist presented here associated with common names and conservation status data is a step towards improving fishery management activities in the Brazilian central coast. Although the use of common names is an important part of the fishery communities culture, the exact identification of exploited fish resources may help to solve doubts about impacts of local and global fisheries. Future researches about regional catches of target fishes and fishing grounds are necessary to better understanding of fisheries dynamics Brazilian central coast. We also emphasize the need to return to the Fisheries Monitoring Program, both state and national.

Acknowledgments - The authors gratefully acknowledge the reviewers and the editor for the reviews and suggestions to improve the paper; to fishers of the Espírito Santo state for their invaluable cooperation; to Capes ( $\mathrm{n}^{\mathrm{o}} 88882315888 / 2019-1$ - financing code, 001- for the granting of a post-doc scholarship of the author JMF).

\section{REFERENCES}

Anticamara, J.A.; Watson, R.; Gelchu, A. \& Pauly, D. Global fishing effort (1950-2010): trends, gaps, and implications. Fish. Res., v. 107, p. 131-136, 2011.

Begossi, A. Ecological, cultural, and economic approaches to managing artisanal fisheries. Environ. Dev. Sustain., v. 16, n. 1, p. 5-34, 2014.

Carpenter, K.E. The living marine resources of the Western Central Atlantic, Food and agriculture organization of the United Nations, 1373 p., Rome, 2002.

Carvalho-Filho, A. Peixes: Costa Brasileira. Melro, 320 p., São Paulo. 1999.

Cervigón, F.; Cipriani, R.; Fisher, W.; Garibaldi, L.; Hendrickx, M.; Lemus, A.; Márquez, R.; Poutiers, J.; Robaina, G. \& Rodríquez, B. Guía de campo de las especies comerciales marinas y de aguas salobres de la costa septentrional de Sur América. Fichas FAO de identificación de especies para los fines de la pesca. FAO, 513 p., 1992.

Cheung, W.W.L.; Sarmiento, J.L.; Dunne, J.; Frolicher, T.L.; Lam, V.W.Y. \& Palomares, M.L.D. Shrinking of fishes exacerbates impacts of global ocean changes on marine ecosystems. Nat. Clim. Change, v. 3, p. 254-258, 2012. http://dx.doi.org/10.1038/nclimate1691

Coulthard, S. What does the debate around social wellbeing have to offer sustainable fisheries? Curr. Opin. Environ. Sustain. v. 4, p. 358-363, 2012. http://dx.doi.org/10.1016/j. cosust.2012.06.001

Defeo, O. \& Castilla, J.C. More than one bag for the world fishery crisis and keys for comanagement successes in selected artisanal Latin American shellfisheries. Rev. Fish Biol. Fish., v. 15, p. 265-283, 2005. 
FAO. The State of World Fisheries and Aquaculture: opportunities and challenges, UN Food and Agriculture Organization, Rome, 2014.

Figueiredo, J.L. Manual de peixes marinhos do Sudeste do Brasil. I. Introdução. Cações, raias e quimeras. São Paulo, Brazil, Museu de Zoologia, USP, 104 p., 1977.

Figueiredo, J.L. \& Menezes, N. Manual de peixes marinhos do Sudeste do Brasil. II. Teleostei (1). Museu de Zoologia, USP, 110 p., São Paulo, 1978.

Figueiredo, J.L. \& Menezes, N. Manual de peixes marinhos do sudeste do Brasil. III. Teleostei (2). Museu de Zoologia, USP, 90 p., São Paulo, 1980.

Figueiredo, J.L. \& Menezes, N. Manual de peixes marinhos do Sudeste do Brasil. VI. Teleostei (5). Museu de Zoologia, USP, 116 p., São Paulo, 2000.

Freire, K.M.F. \& Pauly, D. Richness of common names of Brazilian marine fishes and its effect on catch statistics. J. Ethnobiology, v. 25, n. 2, p. 279-296, 2005.

Freire, K.M.F. \& Carvalho-Filho, A. Richness of common names of Brazilian reef fishes. Pan-Am. J. Aquat. Sci., v. 4, n. 2, p. 96-145, 2009.

Freitas-Netto, R. \& Beneditto, A.P.M.D. Diversidade de artefatos da pesca artesanal marinha do Espírito Santo. Biotemas, Florianópolis, v. 20, n. 2, p. 107-119, 2007.

Froese, R. \& Pauly, D. FishBase, World Wide Web electronic publication, 2019. Available at: http:/ / www.fishbase.org. Accessed at: 14 Feb. 2020.

Garcia, S.M. \& Rosenberg, A.A. Food security and marine capture fisheries: characteristics, trends, drivers and future perspectives, Philos. Trans. R. Soc. B., v. 365, p. 2869-2880, 2010. http://dx.doi.org/10.1098/rstb.2010.0171

Hostim-Silva, M. \& Silva, G.S. Boletim estatístico da pesca do Espírito Santo - Ano 2011. 108 p., 2013.

Ibama. Lista Nacional das Espécies de Invertebrados Aquáticos e Peixes Ameaçadas de Extinção e Lista Nacional das Espécies de Invertebrados Aquáticos e Peixes Sobre-explotadas ou Ameaçadas de Sobre-explotação. 2003. Anexos à Instrução Normativa ${ }^{\circ} 5$ do Ministério do Meio Ambiente, de 21/5/2004, Diário Oficial da União, n 102, seção I, de 28/5/2004.

Instituto Chico Mendes de Conservação da Biodiversidade. Livro Vermelho da Fauna Brasileira Ameaçada de Extinção, ICMBio, 4162 p., Brasília, 2018.

Izzo, C.; Doubleday, Z.A.; Grammer, K.L.; Gilmore, H.K.; Alleway, T.C.; Barnes, M.C.; Disspain, A.J.; Giraldo, A.J.; Mazloumi, N. \& Gillanders, B.M. Fish as proxies of ecological and environmental change. Rev. Fish Biol. Fish, v. 26, p. 265-286, 2016. http://doi.org/10.1007/ s11160-016-9424-3

Jacquet, J. \& Pauly, D. Funding priorities: big barriers to small-scale fisheries. Conserv. Biol.:J.Soc.Conserv.Biol.v.22,p.832-835,2008.http://dx.doi.org/10.1111/j.1523-1739.2008.00978.x Martins, A.S.; Olavo, G. \& Costa, P.A.S. A pesca de linha de alto mar realizada por frotas sediadas no Espírito Santo, Brasil, p. 35-55, in Costa, P.A.S.; Martins, A.S. \& Olavo, G. (eds.), Pesca e potenciais de exploração de recursos vivos na região central da Zona Econômica Exclusiva Brasileira. Museu Nacional, Rio de Janeiro, p. 35-55, 2005.

MPA. Ministério do Meio Ambiente. Boletim estatístico da pesca e aquicultura. 2011. Available at: http://www.icmbio.gov.br/cepsul/images/stories/biblioteca/download/estatistica/est_2011_ bol_bra.pdf. 
Menezes, N. A. \& Figueiredo, J.L. Manual de peixes marinhos do Sudeste do Brasil. IV. Teleostei (3). Museu de Zoologia, USP, 96 p., São Paulo, 1980.

Menezes, N. A. \& Figueiredo, J.L. Manual de peixes marinhos do Sudeste do Brasil. V. Teleostei (4). Museu de Zoologia, USP, 105 p., São Paulo, 1985.

Musiello-Fernandes, J.; Vieira, F.V.; Flores, R.M.; Cabral, L. \& Zappes, C.A. Pesca artesanal e as interferências sobre a atividade na mesorregião central do Espírito Santo. Bol. Mus. Biol. Mello Leitão, Santa Tereza, v. 41, p. 1-21, 2018.

Nelson, J. S.; Grande, T.C. \& Wilson, M.V.H. Fishes of the world. Wiley \& Sons, Hoboken, 707 p., New Jersey, 2016.

Pauly, D.; Christensen, V.; Guénette, S.; Pitcher, T.J.; Sumaila, U.R.; Walters, C.J.; Watson, R. \& Zeller, D. Towards sustainability in world fisheries, Nature, v. 418, p. 689-695, 2002. http://dx.doi.org/10.1038/nature01017

Pinheiro, H.T. \& Joyeux, J.-C. Pescarias multiespecíficas na região da foz do rio Doce, ES, Brasil: características, problemas e opções para um futuro sustentável. Braz. J. Aquatic Sci. Tech., v. 11, p. 15-23, 2007. DOI: 10.14210/bjast.v11n2.p15-23.

Table I - List of families and species catch in fisheries at Espírito Santo state, Central Brazilian coast, with English, Brazilian (BR) and Local name (ES) and Brazilian conservation status. Species classification follows Nelson (2016). English names are based in Froese and Pauly (2019) and BR common names are based in Carvalho-Filho (1999), Freire and Carvalho-Filho (2009) and Froese and Pauly (2019). The conservation status (CS) of the species was determined following the Brazilian Red Book of the Endangered Species (ICMBio, 2018)

\begin{tabular}{|c|c|c|c|c|c|}
\hline Family & Species & English name & BR common name & ES commom name & CS \\
\hline Ginglymostomatidae & $\begin{array}{l}\text { Ginglymostoma } \\
\text { cirratum }\end{array}$ & Nurse shark & Cação-lixa & Cação-lixa, Lixa & VU \\
\hline Lamnidae & Isurus oxyrinchus & Shortfin mako & Cação-anequim & $\begin{array}{l}\text { Cação-anequim, Anequim, } \\
\text { Cação Aniquim }\end{array}$ & \\
\hline Carcharhinidae & Galeocerdo cuvier & Tiger shark & Tubarão-tigre & Cação-tigre, Tintureiro & \\
\hline Carcharhinidae & Prionace glauca & Blue shark & Cação-azul & $\begin{array}{l}\text { Cação-azul, Cação geleia, } \\
\text { Geleia, Cação mole-mole }\end{array}$ & \\
\hline Elopidae & Elops saurus & Ladyfish & Ubarana & Ubarana, Barana & \\
\hline Pristigasteridae & Pellona harroweri & $\begin{array}{l}\text { American coastal } \\
\text { pellona }\end{array}$ & Sardinha-cascudo & Olhudo, Olhuda & \\
\hline Ariidae & Bagre bagre & Coco sea catfish & Ariaçu & Bagre-bandeira, Mabungo & \\
\hline Ariidae & $\begin{array}{l}\text { Notarius } \\
\text { grandicassis }\end{array}$ & Thomas sea catfish & Bagre-beiçudo & Calafate & \\
\hline Merlucciidae & Merluccius hubbsi & Argentine hake & Merluza & Merluza & \\
\hline Holocentridae & $\begin{array}{l}\text { Holocentrus } \\
\text { adscensionis }\end{array}$ & Squirrelfish & Jaguareçá-açú & $\begin{array}{l}\text { Jaguariça, Juriçá, Jaquariça, } \\
\text { Jiriça }\end{array}$ & \\
\hline Dactylopteridae & $\begin{array}{l}\text { Dactylopterus } \\
\text { volitans }\end{array}$ & Flying gurnard & Coió & Falso-voador, Voador & \\
\hline Triglidae & Prionotus punctatus & Bluewingsearobin & $\begin{array}{l}\text { Cabrinha Santo } \\
\text { Antônio }\end{array}$ & Cabrinha & \\
\hline Centropomidae & $\begin{array}{l}\text { Centropomus } \\
\text { undecimalis }\end{array}$ & Common snook & Robalo-verdadeiro & $\begin{array}{l}\text { Robalo, Robalo-flecha, } \\
\text { Robalão }\end{array}$ & \\
\hline Centropomidae & $\begin{array}{l}\text { Centropomus } \\
\text { parallelus }\end{array}$ & Fat snook & Robalo-peba & $\begin{array}{l}\text { Robalo, Robalo-flecha, } \\
\text { Robalão }\end{array}$ & \\
\hline Serranidae & $\begin{array}{l}\text { Dermatolepis } \\
\text { inermis }\end{array}$ & Marbled grouper & Piranema-pintada & Gostosa & \\
\hline
\end{tabular}


(continuation Table 1)

\begin{tabular}{|c|c|c|c|c|c|}
\hline Family & Species & English name & BR common name & ES commom name & CS \\
\hline Serranidae & $\begin{array}{l}\text { Diplectrum } \\
\text { formosum }\end{array}$ & Sand perch & $\begin{array}{l}\text { Michole de areia } \\
\text { listrado }\end{array}$ & Michole, Bichole & \\
\hline Serranidae & Mycteroperca bonaci & Black grouper & Badejo-quadrado & Badejo, Badejo-pérola & VU \\
\hline Serranidae & $\begin{array}{l}\text { Mycteroperca } \\
\text { venenosa }\end{array}$ & Yellowfin grouper & Badejo-ferro & $\begin{array}{l}\text { Piragica, Garoupa-piragica, } \\
\text { Pirajica }\end{array}$ & \\
\hline Serranidae & Cephalopholis fulva & Coney & Piraúna & $\begin{array}{l}\text { Catuá, Catuá grande, Catuá } \\
\text { pequeno }\end{array}$ & \\
\hline Serranidae & Epinephelus morio & Red grouper & $\begin{array}{l}\text { Garoupa São } \\
\text { Tomé }\end{array}$ & Garoupa & VU \\
\hline Priacanthidae & $\begin{array}{l}\text { Priacanthus } \\
\text { arenatus }\end{array}$ & Atlantic bigeye & Olho de cão & $\begin{array}{l}\text { Olho de cão, Cacá, Olho de } \\
\text { vidro }\end{array}$ & \\
\hline Malacanthidae & $\begin{array}{l}\text { Malacanthus } \\
\text { plumieri }\end{array}$ & Sand tilefish & Pirá & Bom nome & \\
\hline Malacanthidae & Lopholatilus villarii & Tile fish & Batata & Batata & VU \\
\hline Pomatomidae & Pomatomus saltatrix & Bluefish & Enchova & Anchova, Enchova & \\
\hline Coryphaenidae & $\begin{array}{l}\text { Coryphaena } \\
\text { hippurus }\end{array}$ & $\begin{array}{l}\text { Common } \\
\text { dolphinfish }\end{array}$ & Dourado & $\begin{array}{l}\text { Dourado, Palombeta, } \\
\text { Dourado exportação }\end{array}$ & \\
\hline Rachycentridae & $\begin{array}{l}\text { Rachycentron } \\
\text { canadum }\end{array}$ & Cobia & Bijupirá & Bijupirá & \\
\hline Carangidae & Caranx crysos & Blue runner & $\begin{array}{l}\text { Carapau- } \\
\text { verdadeiro }\end{array}$ & Xixarro, Carapau & \\
\hline Carangidae & Seriola lalandi & $\begin{array}{l}\text { Yellowtail } \\
\text { amberjack }\end{array}$ & Olhete comum & Caranha & \\
\hline Carangidae & Seriola dumerili & Greater amberjack & Olhete & Olho de boi & \\
\hline Carangidae & Selene setapinnis & Atlantic moonfish & Galo & Peixe-galo, Galo & \\
\hline Carangidae & Selene browni & Caribbean moonfish & Galo de recife & Peixe-galo, Galo & \\
\hline Carangidae & Oligoplites saliens & Castin leatherjacket & Guaivira-salteira & Guaivira, Guaibira, Goibira, & \\
\hline Lutjanidae & Lutjanus analis & Mutton snapper & Cioba & Cirioba & \\
\hline Lutjanidae & Lutjanus synagris & Lane snapper & Ariocó & $\begin{array}{l}\text { Ariacó, Griacó, Areoco, } \\
\text { Greaco, Aroiocô, Oriocó, }\end{array}$ & \\
\hline Lutjanidae & Lutjanus jocu & Dog snapper & Dentão & Dentão & \\
\hline Lutjanidae & Lutjanus vivanus & Silk snapper & $\begin{array}{l}\text { Vermelho olho } \\
\text { amarelo }\end{array}$ & Vermelho papa terra & \\
\hline Lutjanidae & Ocyurus chrysurus & Yellowtail snapper & Guaiúba & $\begin{array}{l}\text { Cioba, Saeoba, Cioba } \\
\text { exportação }\end{array}$ & \\
\hline Lutjanidae & $\begin{array}{l}\text { Rhomboplites } \\
\text { aurorubens }\end{array}$ & Vermilion snapper & Realito & Realito, Mulata & \\
\hline Lobotidae & $\begin{array}{l}\text { Lobotes } \\
\text { surinamensis }\end{array}$ & Tripletail & Prejereba & Dorminhoco & \\
\hline Gerreidae & Diapterus auratus & Irish mojarra & Carapeba branca & Carapeba, Carapeba fraca & \\
\hline Gerreidae & Diapterus rhombeus & Caitipamojarra & Caratingaitê & Carapeba, Carapeba fraca & \\
\hline Haemulidae & $\begin{array}{l}\text { Haemulon } \\
\text { steindachneri }\end{array}$ & Chere-chere grunt & $\begin{array}{l}\text { Cocoroca boca } \\
\text { larga }\end{array}$ & Coró-coró & \\
\hline Haemulidae & Haemulon plumierii & White grunt & Biquara & $\begin{array}{l}\text { Biquara, Boca de velha, } \\
\text { Bicuara, Bicuara, Bricuaria, } \\
\text { Bicoara, Coara, Abiguara }\end{array}$ & \\
\hline Haemulidae & Conodon nobilis & Barred grunt & Canarinho & Roncador & \\
\hline Haemulidae & $\begin{array}{l}\text { Anisotremus } \\
\text { virginicus }\end{array}$ & Porkfish & Frade & Sargofita & \\
\hline Haemulidae & $\begin{array}{l}\text { Anisotremus } \\
\text { moricandi }\end{array}$ & Brownstriped grunt & Fumeiro & Sargo & \\
\hline
\end{tabular}


(continuation Table 1)

\begin{tabular}{|c|c|c|c|c|c|}
\hline Family & Species & English name & BR common name & ES commom name & CS \\
\hline Haemulidae & $\begin{array}{l}\text { Anisotremus } \\
\text { surinamensis }\end{array}$ & Black margate & Sargo-verdadeiro & Sargo & \\
\hline Sparidae & Pagrus pagrus & Red porgy & Pargorosa & $\begin{array}{l}\text { Pargo, Pargo rosa, Pargo } \\
\text { legítimo, Pargo vermelho, } \\
\text { Calunga }\end{array}$ & \\
\hline Sparidae & Calamus pennatula & Pluma porgy & $\begin{array}{l}\text { Peixe pena } \\
\text { amarelo }\end{array}$ & Pargopena & \\
\hline Polynemidae & $\begin{array}{l}\text { Polydactylus } \\
\text { virginicus }\end{array}$ & Barbu & Parati-barbudo & Barbubo & \\
\hline Polynemidae & $\begin{array}{l}\text { Polydactylus } \\
\text { oligodon }\end{array}$ & Littlescale threadfin & Parati-barbudo & Barbubo & \\
\hline Sciaenidae & Larimus breviceps & Shorthead drum & Oveva & Cangoa prata & \\
\hline Sciaenidae & Nebris microps & Smalleye croaker & Banana & Pescada, Pescadinha & \\
\hline Sciaenidae & $\begin{array}{l}\text { Isopisthus } \\
\text { parvipinnis }\end{array}$ & Bigtooth corvina & Pescadinha & Pescada, Pescadinha & \\
\hline Sciaenidae & Macrodon ancylodon & King weakfish & Pescada dentuça & Pescada & \\
\hline Sciaenidae & $\begin{array}{l}\text { Cynoscion } \\
\text { jamaicensis }\end{array}$ & Jamaica weakfish & Goete & $\begin{array}{l}\text { Pescada, Pescadinha, } \\
\text { Pescadinha Maria de Araújo }\end{array}$ & \\
\hline Sciaenidae & Cynoscion virescens & Green weakfish & Pescada branca & Pescada, Pescadinha & \\
\hline Sciaenidae & $\begin{array}{l}\text { Cynoscion } \\
\text { microlepidotus }\end{array}$ & Smallscale weakfish & Dente-de-cão & Pescada, Pescadinha & \\
\hline Sciaenidae & $\begin{array}{l}\text { Menticirrhus } \\
\text { americanus }\end{array}$ & $\begin{array}{l}\text { Southern } \\
\text { kingcroaker }\end{array}$ & Papa-terra & $\begin{array}{l}\text { Papa-terra, Pé de banco, } \\
\text { Betara }\end{array}$ & \\
\hline Sciaenidae & $\begin{array}{l}\text { Menticirrhus } \\
\text { littoralis }\end{array}$ & Gulf kingcroaker & Betara & Papa-terra, Betara & \\
\hline Sciaenidae & $\begin{array}{l}\text { Ctenosciaena } \\
\text { gracilicirrhus }\end{array}$ & Barbel drum & Goretê & $\begin{array}{l}\text { Fofa, Fofa papel, Cabeça } \\
\text { dura }\end{array}$ & \\
\hline Sciaenidae & $\begin{array}{l}\text { Micropogonias } \\
\text { furnieri }\end{array}$ & Whitemouth croaker & Corvina & $\begin{array}{l}\text { Corvina, Tararaca, Corvina } \\
\text { preta, Corvina fraca }\end{array}$ & \\
\hline Sciaenidae & $\begin{array}{l}\text { Paralonchurus } \\
\text { brasiliensis }\end{array}$ & Banded croaker & Maria Luiza & Roncador do norte & \\
\hline Mullidae & $\begin{array}{l}\text { Pseudupeneus } \\
\text { maculatus }\end{array}$ & Spotted goatfish & $\begin{array}{l}\text { Saramonete } \\
\text { pintado }\end{array}$ & Trilha & \\
\hline Labridae & Bodianus pulchellus & Spotfin hogfish & Papagaio azul & Sabonete & \\
\hline Labridae & Bodianus rufus & Spanish hogfish & $\begin{array}{l}\text { Budião papagaio } \\
\text { verdadeiro }\end{array}$ & Sabonete & \\
\hline Labridae & $\begin{array}{l}\text { Halichoeres } \\
\text { dimidiatus }\end{array}$ & Yellowcheek wrasse & Budião azul & Sabonete & \\
\hline Scaridae & Scarus trispinosus & $\begin{array}{l}\text { Greenback } \\
\text { parrotfish }\end{array}$ & Papagaio azul & Budião & $\mathrm{EN}$ \\
\hline Pinguipedidae & $\begin{array}{l}\text { Pseudopercis } \\
\text { semifasciatus }\end{array}$ & $\begin{array}{l}\text { Argentinian } \\
\text { sandperch }\end{array}$ & Namorado & Namorado & \\
\hline Pinguipedidae & $\begin{array}{l}\text { Pseudopercis } \\
\text { numida }\end{array}$ & $\begin{array}{l}\text { Namorado } \\
\text { sandperch }\end{array}$ & $\begin{array}{l}\text { Namorado } \\
\text { verdadeiro }\end{array}$ & Namorado & \\
\hline Gempylidae & Ruvettus pretiosos & Oilfish & Enchova preta & Peixe-prego, Prego & \\
\hline Gempylidae & $\begin{array}{l}\text { Lepidocybium } \\
\text { flavobrunneum }\end{array}$ & Escolar & Escolar & Peixerato & \\
\hline Trichiuridae & Trichiurus lepturus & Largehead hairtail & Espada & Peixe-espada, Espada & \\
\hline Scombridae & Scomber japonicus & Chub mackerel & Cavalinha & Cavalinha & \\
\hline Scombridae & $\begin{array}{l}\text { Euthynnus } \\
\text { alletteratus }\end{array}$ & Little tunny & Bonito cachorro & Gaiado, Galhado & \\
\hline Scombridae & Katsuwonus pelamis & Skipjack tuna & Bonito listrado & Bonito listrado & \\
\hline Scombridae & Thunnus alalunga & Albacore & Atum voador & $\begin{array}{l}\text { Albacora-branca, Atum } \\
\text { voador }\end{array}$ & \\
\hline
\end{tabular}


(continuation Table 1)

\begin{tabular}{|c|c|c|c|c|c|}
\hline Family & Species & English name & BR common name & ES commom name & CS \\
\hline Scombridae & Thunnus obesus & Bigeye tuna & $\begin{array}{l}\text { Albacora- } \\
\text { bandolim }\end{array}$ & $\begin{array}{l}\text { Albacora-bandolim, Atum } \\
\text { bate, Atum bati }\end{array}$ & \\
\hline Scombridae & Thunnus albacares & Yellowfin tuna & Albacora-laje & $\begin{array}{l}\text { Albacora-laje, Atum galha } \\
\text { amarela }\end{array}$ & \\
\hline Scombridae & Thunnus atlanticus & Blackfin tuna & Albacorinha & Atum-atlântico, Pula-pula & \\
\hline Scombridae & $\begin{array}{l}\text { Scomberomorus } \\
\text { cavalla }\end{array}$ & King mackerel & Cavala & $\begin{array}{l}\text { Sarda, Sarda branca, Sarda } \\
\text { sororó, Sororoca, }\end{array}$ & \\
\hline Scombridae & $\begin{array}{l}\text { Scomberomorus } \\
\text { brasiliensis }\end{array}$ & $\begin{array}{l}\text { Serra Spanish } \\
\text { mackerel }\end{array}$ & Serra & $\begin{array}{l}\text { Sarda, Sarda branca, Sarda } \\
\text { sororó, Sororoca, }\end{array}$ & \\
\hline Scombridae & $\begin{array}{l}\text { Acanthocybium } \\
\text { solandri }\end{array}$ & Wahoo & Cavala aipim & $\begin{array}{l}\text { Cavala, Cavala aimpim, } \\
\text { Sarda cavala, Sarda aipim, } \\
\text { Sarda exportação }\end{array}$ & \\
\hline Stromateidae & Peprilus paru & $\begin{array}{l}\text { American } \\
\text { harvestfish }\end{array}$ & Gordinho & Gordinho & \\
\hline Xiphiidae & Xiphias gladius & Swordfish & Espadarte & Espadarte, Meca & \\
\hline Paralichthyidae & $\begin{array}{l}\text { Paralichthys } \\
\text { patagonicus }\end{array}$ & Patagonian flounder & Linguado & Linguado & \\
\hline Balistidae & Balistes vetula & Queen triggerfish & Cangulo-rei & Peroá preta & \\
\hline Balistidae & Balistes capriscus & Grey triggerfish & $\begin{array}{l}\text { Peixe porco } \\
\text { verdadeiro }\end{array}$ & Peroá, Peroá branco & \\
\hline Monacanthidae & Aluterus monoceros & $\begin{array}{l}\text { Unicorn } \\
\text { leatherjacket filefish }\end{array}$ & Gudunho comum & $\begin{array}{l}\text { Peroá cação, Peroá leste, } \\
\text { Peroá liso, Peroá chinelo, } \\
\text { Peroá lanceta, Peroá antena }\end{array}$ & \\
\hline Tetraodontidae & $\begin{array}{l}\text { Lagocephalus } \\
\text { laevigatus }\end{array}$ & Smooth puffer & Baiacu & Baiacu-arara & \\
\hline
\end{tabular}

